\title{
Syntheses and structural characterization of new dithiophosphinato cadmium complexes
}

\author{
ERTUĞRUL GAZİ SAĞLAM ${ }^{\mathrm{a}}$, NURCAN ACAR ${ }^{\mathrm{b}, *}$, YASEMIN SÜZEN $^{\mathrm{c}}$, \\ BERLINE MOUGANG-SOUMÉ ${ }^{\mathrm{d}}$ and TUNCER HÖKELEK ${ }^{\mathrm{e}}$ \\ ${ }^{a}$ Department of Chemistry, Bozok University, 66900, Yozgat, Turkey \\ ${ }^{b}$ Department of Chemistry, Ankara University, 06100 Tandoğan, Ankara, Turkey \\ ${ }^{c}$ Department of Chemistry, Anadolu University, 26470 Yenibağlar, Eskişehir, Turkey \\ ${ }^{\mathrm{d}}$ Department of Chemistry, Université de Montréal, Montréal, Québec, Canada \\ e Department of Physics, Hacettepe University, 06800 Beytepe, Ankara, Turkey \\ e-mail: saglameg@gmail.com; nurcanacar97@gmail.com; ysuzen@gmail.com; djanbe@yahoo.fr; \\ merzifon@hacettepe.edu.tr
}

MS received 27 April 2015; revised 27 June 2015; accepted 29 June 2015

\begin{abstract}
New cadmium complexes of 4-methoxyphenyl dithiophosphinic acids, $\mathrm{H}_{3} \mathrm{CO}-\mathrm{C}_{6} \mathrm{H}_{4}-(\mathrm{R}) \mathrm{PS}_{2} \mathrm{H}$ were prepared. The five dithiophosphinato ligands (L) involved were of the general structure $\mathrm{H}_{3} \mathrm{CO}-\mathrm{C}_{6} \mathrm{H}_{4}-(\mathrm{R}) \mathrm{PS}_{2}^{-}$ with R= 3-methylbutyl, (L1); n-butyl, (L2); 2-methylpropyl, (L3); 1-methylpropyl, (L4) and 2-propyl, (L5). To the best of our knowledge, this is the first report on the preparation and characterization of the n-butylderivative. The acid forms of the ligands were obtained by treatment of the Lawesson reagent, (LR) [2,4-bis(4methoxyphenyl)-1,3,2,4-dithiadiphosphetane-2,4-disulfide] with the corresponding Grignard reagent in dry diethylether. The acids formed were transformed into easily crystallizable ammonium salts $\left(\mathrm{NH}_{4} \mathrm{~L}\right)$ for purification. These salts were treated with $\mathrm{CdCl}_{2}$ in ethanol at room temperature to produce the bis-dithiophosphinato cadmium complexes $\left(\left[\mathrm{Cd}(\mathrm{L})_{2}\right]_{2}\right)$ exclusively. The structures of the complexes were elucidated by elemental analysis, MS, FTIR and Raman spectroscopy techniques as well as ${ }^{1} \mathrm{H}-,{ }^{13} \mathrm{C}$ - and ${ }^{31} \mathrm{P}$ - NMR. The crystal structures of $\left[\mathrm{Cd}(\mathrm{L} 1)_{2}\right]_{2}$ and $\left[\mathrm{Cd}(\mathrm{L} 2)_{2}\right]_{2}$ were also studied as examples. X-ray studies confirmed the nonplanar, fourcoordination geometry of the complexes and indicate that electron delocalization prevails in the $\mathrm{PS}_{2}^{-}$moiety of the dithiophosphinato groups.
\end{abstract}

Keywords. Cadmium dithiophosphinato complexes; Thio-Phosphorus Complexes; Dithiophosphinic acid; Phosphinodithioic acid; X-ray Structure.

\section{Introduction}

Dithiophosphoric acids, $(\mathrm{OR})_{2} \mathrm{P}(\mathrm{S}) \mathrm{SH}$; dithiophosphonic acids, $(\mathrm{OR})(\mathrm{R}) \mathrm{P}(\mathrm{S}) \mathrm{SH}$ and dithiophosphinic acids, $(\mathrm{R})_{2} \mathrm{P}(\mathrm{S}) \mathrm{SH}$, as well as their metal complexes are in current use in rubber vulcanization, ${ }^{1}$ lubricating oils $^{2}$ and pesticides. ${ }^{3}$ Tin and antimony complexes of some dithiophosphinic acids (DTPA) are also of potentially useful as chemotherapy agents in the treatment of some cancers. ${ }^{4}$ In addition, some DTPA- ${ }^{99} \mathrm{Tc}$ complexes have been introduced as brain imaging agents in radiography ${ }^{5}$ and cephalosporin-type chemicals containing DTPA moieties have been suggested as antibiotics in clinical medicine. ${ }^{6}$ The commercial sequestering agent CYANEX 301, (bis(2,4,4-trimethylpentyl)dithiophosphinic acid) is in current use for the extraction metals from ores. ${ }^{7}$ The same agent is

*For correspondence also employed in heavy metal removal from industrial wastes. $^{8}$

The synthesis of DTPA-type compounds is relatively more tedious compared to the synthesis of other dithiophosphorus acids. ${ }^{9}$ Coupled with their disagreeable odour and the difficulty in obtaining the starting reagents, these compounds have attracted comparatively less attention in the past. ${ }^{10}$ Various routes have been developed for the DTPA synthesis, ${ }^{11}$ the most general one being the addition reaction of Grignard reagents with perthiophosphonic acid anhydrides such as Lawesson reagent, (LR). The DTPAs formed were further reacted with ammonia to form the easily crystallizable ammonium salts, facilitating a quick refinement of the DTPA. The ammonium salt is stable and can easily be converted to complexes when desired. ${ }^{12}$

Depending on the identity of the metal cation, the coordination number of the DTPA complex changes. Most of the complexes generally display a four- or 
six-coordination. ${ }^{13}$ The nickel(II) complexes are exclusively mono-nuclear and generally of a square-planar topology as is the case with other soft ligands; whereas, DTPA complexes of manganese(II), cobalt(II), Zinc(II) and cadmium(II) are known to display four coordinated, dimeric structures. ${ }^{14}$ In the case of the latter complexes, $\mathrm{S}$ atoms of the $\mathrm{PS}_{2}^{-}$group are known to act as singly-bonded and also as bridging ligands.

X-ray crystallographic studies on the crystal structures ditihophosphinato complexes of group 12 metals, namely, zinc (II), cadmium (II) and mercury (II), are scarce. These dimeric complexes display a chair conformation although some complexes are known to display a boat conformation. ${ }^{14 c, 15}$

\section{Experimental}

\subsection{Materials and Methods}

Analytical-grade LR, 3-methylbutyl bromide, n-butyl bromide, 2-methylpropyl bromide, 1-methylpropyl bromide and 2-propyl bromide were purchased from Merck and used without further purification. Benzene, chloroform, ethanol, diethyl ether, $\mathrm{CdCl}_{2}$ were purchased from Merck. Benzene and diethyl ether were distilled and dried before use. $\mathrm{NH}_{4} \mathrm{~L} 1, \mathrm{NH}_{4} \mathrm{~L} 2, \mathrm{NH}_{4} \mathrm{~L} 3$, $\mathrm{NH}_{4} \mathrm{~L} 4$ and $\mathrm{NH}_{4} \mathrm{~L} 5$ were prepared according to the literature. $^{12 \mathrm{a}, 16,17}$

The LC/MS spectra were recorded with a Waters Micromass ZQ connected with Waters Alliance HPLC, using $\mathrm{ESI}(+)$ ionization and C-18 column. Melting points were measured with a Gallenkamp apparatus using a capillary tube. ${ }^{1} \mathrm{H}-,{ }^{13} \mathrm{C}\left\{{ }^{1} \mathrm{H}\right\}-$ and ${ }^{31} \mathrm{P}\left\{{ }^{1} \mathrm{H}\right\}$ - spectra were recorded with a Varian Mercury (Agilent) $400 \mathrm{MHz}$ FT spectrometer in $\mathrm{CDCl}_{3} \mathrm{SiMe}_{4}\left({ }^{1} \mathrm{H}\right.$, $\left.{ }^{13} \mathrm{C}\right)$ and $85 \% \mathrm{H}_{3} \mathrm{PO}_{4}\left({ }^{31} \mathrm{P}\right)$ were used as standards. IR spectra were recorded on a Perkin Elmer Spectrum 400 FTIR spectrophotometer $\left(200-4000 \mathrm{~cm}^{-1}\right)$ and are reported in $\mathrm{cm}^{-1}$ units. All Raman spectra were measured in the range of $4000-100 \mathrm{~cm}^{-1}$, at room temperature, using a Renishaw in-Via Raman microscope, equipped with Peltier-cooled CCD detectors $\left(-70^{\circ} \mathrm{C}\right)$. For Raman microscopy, a 50X objective was usually used and all the spectra were excited by the 785 line of a diode laser. Microanalyses were performed using a LECO CHNS-932 C elemental analyzer.

\subsection{Preparation and structural data}

2.2.1 Ammonium n-butyl(4-methoxyphenyl)dithiophosphinate, $\mathrm{NH}_{4} \mathrm{L2}$ : The method of preparation was the same as described in the literature. ${ }^{16}$ : Yield: $1.52 \mathrm{~g}$
(55\%). White colour. M.P. $179-180^{\circ} \mathrm{C}$. NMR spectroscopic data and mass data are as follows: ${ }^{1} \mathrm{H}$ NMR (in $\left.\mathrm{D}_{2} \mathrm{O}\right): \delta=0.66\left(\mathrm{t},{ }^{3} J_{\mathrm{HH}}=7.29 \mathrm{~Hz}, 6 \mathrm{H},-\mathrm{C} 9 \underline{\mathrm{H}}\right) ; 1.14(\mathrm{~m}$, $2 \mathrm{H},-\mathrm{C} 7 \underline{\mathrm{H}}) ; 1.27$ (m, 2H, -C8파); $2.06(\mathrm{~m}, 2 \mathrm{H},-\mathrm{C} 6 \underline{\mathrm{H}})$; $3.70\left(\mathrm{~s}, 3 \mathrm{H}, \mathrm{OC}_{3}\right) ; 6.89\left(\mathrm{~A}-\right.$ part of $\mathrm{AA}^{\prime} \mathrm{MM}^{\prime} \mathrm{X},{ }^{4} J_{\mathrm{PH}}=$ $\left.2.13 \mathrm{~Hz}\left(J_{\mathrm{AX}}\right), N=\mathrm{J}_{\mathrm{AM}}+\mathrm{J}_{\mathrm{AM}^{\prime}}=8.90 \mathrm{~Hz}, 2 \mathrm{H}, m-\mathrm{H}\right)$; 7.80 (M-part of AA'MM'X, ${ }^{3} J_{\mathrm{PH}}=12.60 \mathrm{~Hz}\left(J_{\mathrm{MX}}\right)$, $N=8.90 \mathrm{~Hz}, 2 \mathrm{H}, o-\mathrm{H}) \cdot{ }^{13} \mathrm{C}-\mathrm{NMR}\left(\mathrm{ppm}\right.$, in $\left.\mathrm{D}_{2} \mathrm{O}\right): \delta=$ 12.9 (s, - $\underline{\mathrm{C}} 9) ; 22.8\left(\mathrm{~d},{ }^{2} J_{\mathrm{P}-\mathrm{C}}=17.86 \mathrm{~Hz}, \underline{\mathrm{C}} 7\right) ; 25.9$ $\left(\mathrm{d},{ }^{3} J_{\mathrm{P}-\mathrm{C}}=4.1 \mathrm{~Hz}, \underline{\mathrm{C}} 8\right) ; 43.6\left(\mathrm{~d}, J_{\mathrm{P}-\mathrm{C}}=56.1 \mathrm{~Hz}, \mathrm{P}-\right.$ C6); $55.4\left(\mathrm{~s}, \mathrm{O}-\mathrm{C}_{5} \mathrm{H}_{3}\right) ; 113.5\left(\mathrm{~d},{ }^{3} J_{\mathrm{P}-\mathrm{C}}=13.3 \mathrm{~Hz}, \mathrm{Ar}-\right.$ $\left.\mathrm{C}_{\text {meta }}\right) ; 131.8\left(\mathrm{~d}, J_{\mathrm{P}-\mathrm{C}}=80.5 \mathrm{~Hz}, \mathrm{P}-\mathrm{C}_{\text {arom }}\right) ; 131.7(\mathrm{~d}$, ${ }^{2} J_{\mathrm{P}-\mathrm{C}}=13.3 \mathrm{~Hz}$, Ar-C $\left.\mathrm{C}_{\text {ortho }}\right) ; 163.5\left(\mathrm{~d},{ }^{4} J_{\mathrm{P}-\mathrm{C}}=3.0 \mathrm{~Hz}\right.$, $\mathrm{CH}_{3} \mathrm{O}-\underline{\mathrm{C}}$ ). ${ }^{31} \mathrm{P}-\mathrm{NMR}$ (in $\mathrm{D}_{2} \mathrm{O}$ ): $\delta=63.4$. LC/MS MS: $\mathrm{m} / \mathrm{z} 261.2\left([\mathrm{~L} 2]^{+}, 100 \%\right), 227.2\left(\left[\left(\mathrm{~L} 2-\left(\mathrm{n}-\mathrm{C}_{4} \mathrm{H}_{9}\right)+\mathrm{Na}\right]^{+}\right.\right.$, 49\%), 205.3 ([L2-(n- $\left.\left.\mathrm{C}_{4} \mathrm{H}_{9}\right]^{+}, 11 \%\right)$. Anal. Calcd. for $\mathrm{C}_{11} \mathrm{H}_{20} \mathrm{NOPS}_{2}\left(277.4\right.$ g.mol $\left.{ }^{-1}\right)$ : C, 47.6; H, 7.3; N, 5.1; S, 23.1; found: C, 47.8; H, 7.3; N, 5.2; S, $23.4 \%$.

2.2.2 Preparation of the complexes, $\left[C d(L)_{2}\right]_{2}$ : A solution of $\mathrm{CdCl}_{2},(0.1 \mathrm{~g}, 0.55 \mathrm{mmol})$ in ethanol $(10$ $\mathrm{mL}$ ) was added to an ethanolic solution $(10 \mathrm{~mL})$ of 1.09 mmol ligand $\left(0.32 \mathrm{~g}\right.$, for $\mathrm{NH}_{4} \mathrm{~L} 1 ; 0.30 \mathrm{~g}$, for $\mathrm{NH}_{4} \mathrm{~L} 2$; $0.30 \mathrm{~g}$, for $\mathrm{NH}_{4} \mathrm{~L} 3$ and $\mathrm{NH}_{4} \mathrm{~L} 4 ; 0.29 \mathrm{~g}$, for $\mathrm{NH}_{4} \mathrm{~L} 5$ ). The mixture was stirred overnight at room temperature. The resulting solution was left to stand overnight. The complexes were of colourless, fine crystallic appearance. The crystals were filtered off and recrystallized from chloroform. The numbering scheme for compounds is given in figure 1 . The structural data for the complexes were as follows.

Bis-\{bis-[4-methoxyphenyl(3-methylbutyl)dithiophosphinato]cadmium(II)\}, $\left[\mathrm{Cd}(\mathrm{Ll})_{2}\right]_{2}$ [Yield: $0.59 \mathrm{~g}, 73 \%$ ]. Colourless. M.p. $209-210^{\circ} \mathrm{C}$. ${ }^{1} \mathrm{H}$ NMR (ppm, in $\left.\mathrm{CDCl}_{3}\right): \delta=0.84\left(\mathrm{~d},{ }^{3} J_{\mathrm{HH}}=6.5 \mathrm{~Hz}, 24 \mathrm{H},-\mathrm{C} 9 \mathrm{H}\right)$; $1.44(\mathrm{~m}, 8 \mathrm{H},-\mathrm{C} 8 \mathrm{H}) ; 1.54(\mathrm{~m}, 8 \mathrm{H},-\mathrm{C} 7 \mathrm{H}) ; 2.28(\mathrm{~m}$, $8 \mathrm{H},-\mathrm{C} 6 \mathrm{H}) ; 3.84\left(\mathrm{~s}, 12 \mathrm{H}, \mathrm{OCH}_{3}\right) ; 6.88$ (A-part of $\mathrm{AA}^{\prime} \mathrm{MM}^{\prime} \mathrm{X},{ }^{4} J_{\mathrm{PH}}=2.4 \mathrm{~Hz}\left(J_{\mathrm{AX}}\right), N=\mathrm{J}_{\mathrm{AM}}+\mathrm{J}_{\mathrm{AM}^{\prime}}=$ $8.7 \mathrm{~Hz}, 8 \mathrm{H}, m-\mathrm{H}) ; 7.89\left(\mathrm{M}-\right.$ part of $\mathrm{AA}^{\prime} \mathrm{MM}^{\prime} \mathrm{X},{ }^{3} J_{\mathrm{PH}}=$ $\left.13.4 \mathrm{~Hz}\left(J_{\mathrm{MX}}\right), N=8.7 \mathrm{~Hz}, 8 \mathrm{H}, o-\mathrm{H}\right) .{ }^{13} \mathrm{C}-\mathrm{NMR}(\mathrm{ppm}$, in $\left.\mathrm{CDCl}_{3}\right): \delta=22.2(\mathrm{~s},-\mathrm{C} 9) ; 32.5\left(\mathrm{~d},{ }^{3} J_{\mathrm{P}-\mathrm{C}}=4.3\right.$ $\mathrm{Hz}, \mathrm{C} 8) ; 28.6\left(\mathrm{~d},{ }^{2} J_{\mathrm{P}-\mathrm{C}}=17.5 \mathrm{~Hz}, \mathrm{C} 7\right) ; 41.2(\mathrm{~d}$, $\left.J_{\mathrm{P}-\mathrm{C}}=52.5 \mathrm{~Hz}, \mathrm{P}-\mathrm{C} 6\right) ; 55.3\left(\mathrm{~s}, \mathrm{O}-\mathrm{C} 5 \mathrm{H}_{3}\right) ; 113.7(\mathrm{~d}$, $\left.{ }^{3} J_{\mathrm{P}-\mathrm{C}}=14.1 \mathrm{~Hz}, \mathrm{Ar}-\mathrm{C}_{\text {meta }}\right) ; 126.9\left(\mathrm{~d}, J_{\mathrm{P}-\mathrm{C}}=80.2\right.$ $\mathrm{Hz}, \mathrm{P}-\mathrm{C} 1) ; 132.6\left(\mathrm{~d},{ }^{2} \mathrm{~J}_{\mathrm{P}-\mathrm{C}}=13.0 \mathrm{~Hz}, \mathrm{Ar}_{-\mathrm{C}}\right.$ ortho $)$; $161.9\left(\mathrm{~d},{ }^{4} \mathrm{~J}_{\mathrm{P}-\mathrm{C}}=3.0 \mathrm{~Hz}, \mathrm{CH}_{3} \mathrm{O}-\mathrm{C} 4\right) .{ }^{31} \mathrm{P}-\mathrm{NMR}$ (ppm, in $\mathrm{CDCl}_{3}$ ): $\delta=71.9$. Analysis: Calculated for $\mathrm{C}_{48} \mathrm{H}_{72} \mathrm{Cd}_{2} \mathrm{O}_{8} \mathrm{P}_{4} \mathrm{~S}_{8}\left(1318.3\right.$ g.mol $^{-1}$ ): C, 43.7; H, 5.5; $\mathrm{S}$, 19.5. Found: C, 43.8; H, 5.7; S, 19.6\%. LC/MS: $m / z$ $243.0\left(\left[\mathrm{~L} 1-\left(\mathrm{CH}_{3} \mathrm{O}-\right)\right]^{+}, 100 \%\right), 1045.3\left(\left[\mathrm{Cd}_{2}(\mathrm{~L} 1)_{3}\right]^{+}\right.$, $14 \%)$. 


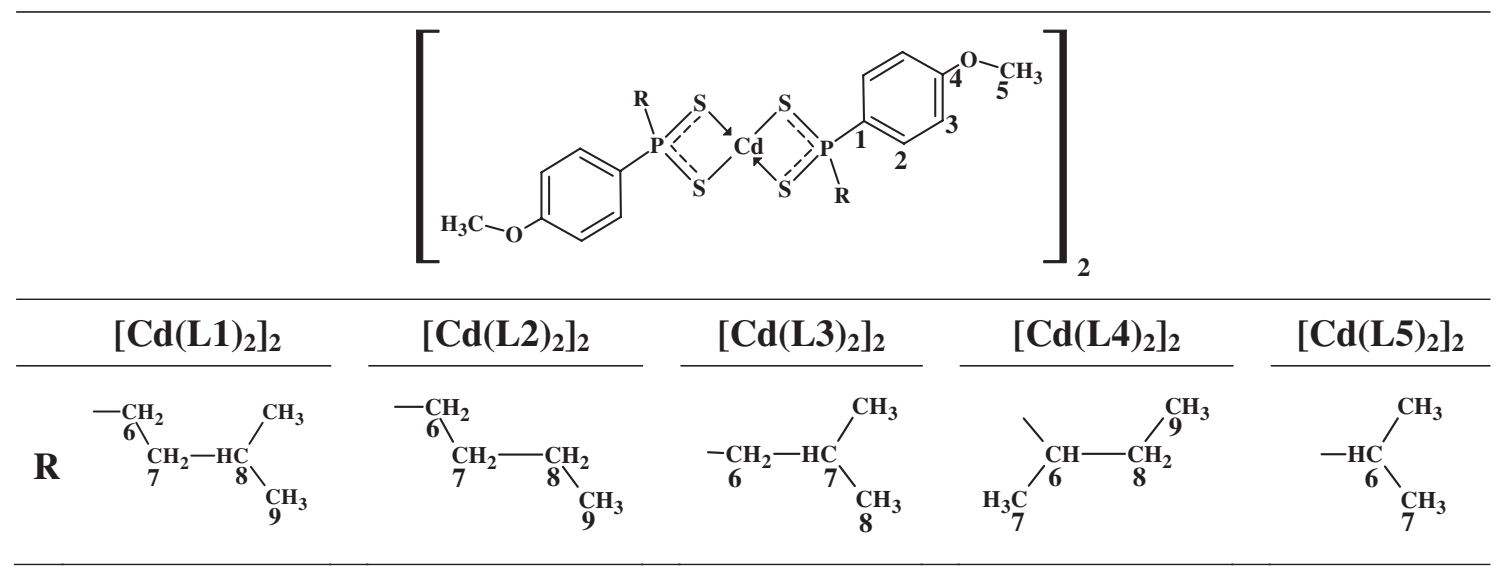

s: singlet; d: doublet; t: triplet; dd: doublet of doublets; m: multiplet.

Figure 1. Numbering scheme for compound.

Bis-\{bis-[n-butyl(4-methoxyphenyl)dithiophosphinato] $\operatorname{cadmium}(I I)\}, \quad\left[C d(L 2)_{2}\right]_{2} \quad$ [Yield: $0.58 \mathrm{~g}, \quad 84 \%$ ]. Colourless. M.p. ${ }^{144-145^{\circ} \mathrm{C} .}{ }^{1} \mathrm{H}$ NMR (ppm, in $\left.\mathrm{CDCl}_{3}\right): \delta=0.84\left(\mathrm{~d},{ }^{3} \mathrm{~J}_{\mathrm{HH}}=7.3 \mathrm{~Hz}, 12 \mathrm{H},-\mathrm{C} 9 \mathrm{H}\right)$; $1.32(\mathrm{~m}, 8 \mathrm{H},-\mathrm{C} 8 \mathrm{H}) ; 1.53(\mathrm{~m}, 8 \mathrm{H},-\mathrm{C} 7 \mathrm{H}) ; 2.28(\mathrm{~m}$, $8 \mathrm{H},-\mathrm{C} 6 \mathrm{H}) ; 3.83\left(\mathrm{~s}, 12 \mathrm{H}, \mathrm{OCH}_{3}\right) ; 6.93$ (A-part of $\mathrm{AA}^{\prime} \mathrm{MM}^{\prime} \mathrm{X},{ }^{4} \mathrm{~J}_{\mathrm{PH}}=2.4 \mathrm{~Hz}\left(\mathrm{~J}_{\mathrm{AX}}\right), \mathrm{N}=\mathrm{J}_{\mathrm{AM}}+\mathrm{J}_{\mathrm{AM}^{\prime}}=$ $8.8 \mathrm{~Hz}, 8 \mathrm{H}, \mathrm{m}-\mathrm{H}) ; 7.89$ (M-part of $\mathrm{AA}^{\prime} \mathrm{MM}^{\prime} \mathrm{X},{ }^{3} \mathrm{~J}_{\mathrm{PH}}=$ $\left.13.4 \mathrm{~Hz}\left(\mathrm{~J}_{\mathrm{MX}}\right), \mathrm{N}=8.8 \mathrm{~Hz}, 8 \mathrm{H}, \mathrm{o}-\mathrm{H}\right) .{ }^{13} \mathrm{C}-\mathrm{NMR}$ (ppm, in $\left.\mathrm{CDCl}_{3}\right): \delta=13.6$ (s, -C9); $25.9\left(\mathrm{~d},{ }^{3} \mathrm{~J}_{\mathrm{P}-\mathrm{C}}=4.2 \mathrm{~Hz}\right.$, $\mathrm{C} 8) ; 23.3\left(\mathrm{~d},{ }^{2} \mathrm{~J}_{\mathrm{P}-\mathrm{C}}=18.6 \mathrm{~Hz}, \mathrm{C} 7\right) ; 42.9\left(\mathrm{~d}, \mathrm{~J}_{\mathrm{P}-\mathrm{C}}=52.3\right.$ $\mathrm{Hz}, \mathrm{P}-\mathrm{C} 6) ; 55.4\left(\mathrm{~s}, \mathrm{O}-\mathrm{C} 5 \mathrm{H}_{3}\right) ; 113.7\left(\mathrm{~d},{ }^{3} \mathrm{~J}_{\mathrm{P}-\mathrm{C}}=14.1\right.$ $\left.\mathrm{Hz}, \mathrm{Ar}_{-} \mathrm{C}_{\text {meta }}\right) ; 127.0\left(\mathrm{~d}, \mathrm{~J}_{\mathrm{P}-\mathrm{C}}=80.3 \mathrm{~Hz}, \mathrm{P}-\mathrm{C} 1\right) ; 132.6$ $\left(\mathrm{d},{ }^{2} \mathrm{~J}_{\mathrm{P}-\mathrm{C}}=13.0 \mathrm{~Hz}\right.$, Ar- $\left.\mathrm{C}_{\text {ortho }}\right) ; 162.0\left(\mathrm{~d},{ }^{4} \mathrm{~J}_{\mathrm{P}-\mathrm{C}}=2.9\right.$ $\left.\mathrm{Hz}, \mathrm{CH}_{3} \mathrm{O}-\mathrm{C} 4\right) .{ }^{31} \mathrm{P}-\mathrm{NMR}$ (ppm, in $\mathrm{CDCl}_{3}$ ): $\delta=71.5$. Analysis: Calculated for $\mathrm{C}_{44} \mathrm{H}_{64} \mathrm{Cd}_{2} \mathrm{O}_{4} \mathrm{P}_{4} \mathrm{~S}_{8}(1262.2 \mathrm{~g}$ $\mathrm{mol}^{-1}$ ): C,41.9; H, 5.1; S, 20.3. Found: C, 42.0; H, 5.3; S, 20.3\%. LC/MS: m/z 228.9 ([L2-( $\left.\left.\left.\mathrm{CH}_{3} \mathrm{O}-\right)\right]^{+}, 100 \%\right)$, $632.9\left(\left[\mathrm{Cd}(\mathrm{L} 2)_{2}\right]^{+}, 7 \%\right), 1002.9\left(\left[\mathrm{Cd}_{2}(\mathrm{~L} 2)_{3}\right]^{+}, 6 \%\right)$, $1265.4\left(\left[\mathrm{Cd}_{2}(\mathrm{~L} 2)_{4}\right]^{+}, 4 \%\right)$.

Bis-\{bis-[4-methoxyphenyl(2-methylpropyl)dithiophosphinato]cadmium(II)\}, $\left[\mathrm{Cd}(\mathrm{L} 3)_{2}\right]_{2}$ [Yield: $0.55 \mathrm{~g}$, 79\%] Colourless. M.p. 199-200 ${ }^{\circ}$ C. ${ }^{1} \mathrm{H}$ NMR (ppm, in $\left.\mathrm{CDCl}_{3}\right): \delta=0.88\left(\mathrm{~d},{ }^{3} J_{\mathrm{HH}}=6.7 \mathrm{~Hz}, 24 \mathrm{H},-\mathrm{C} 8 \mathrm{H}\right)$; $2.11(\mathrm{~m}, 4 \mathrm{H},-\mathrm{C} 7 \mathrm{H}) ; 2.28\left(\mathrm{dd},{ }^{3} J_{\mathrm{HH}}=6.1 \mathrm{~Hz}\right.$, $\left.{ }^{2} J_{\mathrm{P}-\mathrm{H}}=11.9 \mathrm{~Hz}, 8 \mathrm{H},-\mathrm{C} 6 \mathrm{H}\right) ; 3.84\left(\mathrm{~s}, 12 \mathrm{H}, \mathrm{OCH}_{3}\right)$; 6.92 (A-part of $\mathrm{AA}^{\prime} \mathrm{MM} \mathrm{M}^{\prime} \mathrm{X},{ }^{4} J_{\mathrm{PH}}=2.5 \mathrm{~Hz}\left(J_{\mathrm{AX}}\right)$, $\left.N=\mathrm{J}_{\mathrm{AM}}+\mathrm{J}_{\mathrm{AM}^{\prime}}=8.8 \mathrm{~Hz}, 8 \mathrm{H}, m-\mathrm{H}\right) ; 7.91$ (M-part of $\mathrm{AA}^{\prime} \mathrm{MM}^{\prime} \mathrm{X},{ }^{3} J_{\mathrm{PH}}=13.4 \mathrm{~Hz}\left(J_{\mathrm{MX}}\right), N=8.8 \mathrm{~Hz}$, $8 \mathrm{H}, o-\mathrm{H}) .{ }^{13} \mathrm{C}-\mathrm{NMR}\left(\mathrm{ppm}\right.$, in $\left.\mathrm{CDCl}_{3}\right): \delta=25.5(\mathrm{~d}$, $\left.{ }^{3} J_{\mathrm{P}-\mathrm{C}}=3.9 \mathrm{~Hz}, \mathrm{C} 8\right) ; 24.3\left(\mathrm{~d},{ }^{2} J_{\mathrm{P}-\mathrm{C}}=10.0 \mathrm{~Hz}, \mathrm{C} 7\right)$; $42.9\left(\mathrm{~d}, J_{\mathrm{P}-\mathrm{C}}=51.4 \mathrm{~Hz}, \mathrm{P}-\mathrm{C} 6\right) ; 55.4\left(\mathrm{~s}, \mathrm{O}-\mathrm{C} 5 \mathrm{H}_{3}\right)$; $113.7\left(\mathrm{~d},{ }^{3} J_{\mathrm{P}-\mathrm{C}}=14.1 \mathrm{~Hz}\right.$, Ar- $\left.\mathrm{C}_{\text {meta }}\right) ; 127.8\left(\mathrm{~d}, J_{\mathrm{P}-\mathrm{C}}=\right.$ $80.3 \mathrm{~Hz}, \mathrm{P}-\mathrm{C} 1) ; 132.6$ (d, $\left.{ }^{2} J_{\mathrm{P}-\mathrm{C}}=13.1 \mathrm{~Hz}, \mathrm{Ar}-\mathrm{C}_{\text {ortho }}\right)$; $161.9\left(\mathrm{~d},{ }^{4} \mathrm{~J}_{\mathrm{P}-\mathrm{C}}=2.9 \mathrm{~Hz}, \mathrm{CH}_{3} \mathrm{O}-\mathrm{C} 4\right) .{ }^{31} \mathrm{P}-\mathrm{NMR}$ (ppm, in $\left.\mathrm{CDCl}_{3}\right): \delta=70.1$. Analysis: Calculated for $\mathrm{C}_{44} \mathrm{H}_{64} \mathrm{Cd}_{2} \mathrm{O}_{4} \mathrm{P}_{4} \mathrm{~S}_{8}\left(1262.2 \mathrm{~g} \mathrm{~mol}^{-1}\right): \mathrm{C}, 41.9 ; \mathrm{H}, 5.1$; S, 20.3\%. Found: C, 42.0; H, 5.4; S, 20.4\%. LC/MS: $m / z 325.1\left(\left[\left(\left(\mathrm{C}_{6} \mathrm{H}_{5}\right) \mathrm{PS}_{2}\right) \mathrm{Cd}+\mathrm{CH}_{3} \mathrm{CN}\right]^{+}, 100 \%\right), 413.9$ $\left(\left[\mathrm{Cd}(\mathrm{L} 3)+\mathrm{CH}_{3} \mathrm{CN}\right]^{+}, 8 \%\right)$.

Bis-\{bis-[4-methoxyphenyl(1-methylpropyl)dithiophosphinato]cadmium(II)\}, $\left[\mathrm{Cd}(\mathrm{L} 4)_{2}\right]_{2} \quad$ [Yield: $0.56 \mathrm{~g}$, 81\%] Colourless. M.p. 247-248 ${ }^{\circ}$ C. ${ }^{1} \mathrm{H}$ NMR (ppm, in $\left.\mathrm{CDCl}_{3}\right): \delta=0.89\left(\mathrm{t},{ }^{3} \mathrm{~J}_{\mathrm{HH}}=7.3 \mathrm{~Hz}, 12 \mathrm{H},-\mathrm{C} 9 \mathrm{H}\right)$; $1.96(\mathrm{~m}, 8 \mathrm{H},-\mathrm{C} 8 \mathrm{H}) ; 1.16\left(\mathrm{~m},{ }^{3} \mathrm{~J}_{\mathrm{HH}}=6.86 \mathrm{~Hz},{ }^{2} \mathrm{~J}_{\mathrm{P}-\mathrm{H}}=\right.$ $21.94 \mathrm{~Hz}, 16 \mathrm{H}, \mathrm{C} 6-\mathrm{H}$ and $\mathrm{C} 7-\mathrm{H}$ adjacent); 1.16 (m, $16 \mathrm{H}, \mathrm{C} 6-\mathrm{H}$ and $\mathrm{C} 7-\mathrm{H}$ adjacent); 3.84 (s, $\left.12 \mathrm{H}, \mathrm{OCH}_{3}\right)$; 6.92 (A-part of $\mathrm{AA}^{\prime} \mathrm{MM} \mathrm{M}^{\prime} \mathrm{X},{ }^{4} \mathrm{~J}_{\mathrm{PH}}=2.4 \mathrm{~Hz}\left(\mathrm{~J}_{\mathrm{AX}}\right)$, $\mathrm{N}=\mathrm{J}_{\mathrm{AM}}+\mathrm{J}_{\mathrm{AM}^{\prime}}=8.9 \mathrm{~Hz}, 8 \mathrm{H}, \mathrm{m}-\mathrm{H}$ ); 7.87 (M-part of $\mathrm{AA}^{\prime} \mathrm{MM}^{\prime} \mathrm{X},{ }^{3} \mathrm{~J}_{\mathrm{PH}}=12.7 \mathrm{~Hz}\left(\mathrm{~J}_{\mathrm{MX}}\right), \mathrm{N}=8.9 \mathrm{~Hz}, 8 \mathrm{H}$, $\mathrm{o}-\mathrm{H}$ ). ${ }^{13} \mathrm{C}-\mathrm{NMR}$ (ppm, in $\mathrm{CDCl}_{3}$ ): $\delta=12.3$ (s, -C9); 23.2 (s, C8); $12.3\left(\mathrm{~d},{ }^{2} \mathrm{~J}_{\mathrm{P}-\mathrm{C}}=16.8 \mathrm{~Hz}, \mathrm{C} 7\right) ; 45.3$ (d, $\left.\mathrm{J}_{\mathrm{P}-\mathrm{C}}=50.3 \mathrm{~Hz}, \mathrm{P}-\mathrm{C} 6\right) ; 55.3\left(\mathrm{~s}, \mathrm{O}-\mathrm{C} \mathrm{H}_{3}\right) ; 113.4(\mathrm{~d}$, ${ }^{3} \mathrm{~J}_{\mathrm{P}-\mathrm{C}}=13.7 \mathrm{~Hz}$, Ar- $\left.\mathrm{C}_{\text {meta }}\right) ; 126.6\left(\mathrm{~d}, \mathrm{~J}_{\mathrm{P}-\mathrm{C}}=77.3\right.$ $\mathrm{Hz}, \mathrm{P}-\mathrm{C} 1) ; 133.4\left(\mathrm{~d},{ }^{2} \mathrm{~J}_{\mathrm{P}-\mathrm{C}}=13.7 \mathrm{~Hz}\right.$, Ar-C $\left.{ }_{\text {ortho }}\right)$; $161.9\left(\mathrm{~d},{ }^{4} \mathrm{~J}_{\mathrm{P}-\mathrm{C}}=2.9 \mathrm{~Hz}, \mathrm{CH}_{3} \mathrm{O}-\mathrm{C} 4\right) .{ }^{31} \mathrm{P}-\mathrm{NMR}$ (ppm, in $\mathrm{CDCl}_{3}$ ): $\delta=82.5$. Analysis: Calculated for $\mathrm{C}_{44} \mathrm{H}_{64} \mathrm{Cd}_{2} \mathrm{O}_{4} \mathrm{P}_{4} \mathrm{~S}_{8}$ (1262.2 $\left.\mathrm{g} \mathrm{mol}^{-1}\right)$ : C,41.9; H, 5.1; S, 20.3\%. Found: C, 42.1; H, 5.2; S, 20.5\%. LC/MS: $\mathrm{m} / \mathrm{z} 391.1 \quad\left(\left[\mathrm{Cd}_{2} \mathrm{P}_{2} \mathrm{~S}_{2}+\mathrm{CH}_{3} \mathrm{CN}\right]^{+}, \quad 100 \%\right), 413.1$ $\left(\left[\mathrm{Cd}_{2} \mathrm{P}_{2} \mathrm{~S}_{4}+\mathrm{H}\right]^{+}, 77 \%\right), 1001.0\left(\left[\mathrm{Cd}_{2}(\mathrm{~L} 4)_{3}\right]^{+}, 4 \%\right)$, $1265.3\left(\left[\mathrm{Cd}_{2}(\mathrm{~L} 4)_{4}\right]^{+}, 11 \%\right)$.

Bis-\{bis[iso-propyl(4-methoxyphenyl)dithiophosphinato]cadmium(II)\} [Cd(L5) $]_{2}$ [Yield: $0.62 \mathrm{~g}, 94 \%$ ] Colourless. M.p. ${ }^{129-130}{ }^{\circ} \mathrm{C} .{ }^{1} \mathrm{H}$ NMR (ppm, in $\left.\mathrm{CDCl}_{3}\right): \delta=1.12\left(\mathrm{t},{ }^{3} J_{\mathrm{PH}}=21.2 \mathrm{~Hz}, 24 \mathrm{H},-\mathrm{C} 7 \mathrm{H}\right)$; $2.25(\mathrm{~m}, 4 \mathrm{H},-\mathrm{C} 7 \mathrm{H}) ; 3.84\left(\mathrm{~s}, 12 \mathrm{H}, \mathrm{OCH}_{3}\right) ; 6.92(\mathrm{~A}-$ part of $\mathrm{AA}^{\prime} \mathrm{MM}^{\prime} \mathrm{X},{ }^{4} \mathrm{~J}_{\mathrm{PH}}=2.4 \mathrm{~Hz}\left(J_{\mathrm{AX}}\right), N=\mathrm{J}_{\mathrm{AM}}+$ $\left.\mathrm{J}_{\mathrm{AM}^{\prime}}=8.9 \mathrm{~Hz}, 8 \mathrm{H}, m-\mathrm{H}\right) ; 7.9$ (M-part of $\mathrm{AA}^{\prime} \mathrm{MM}^{\prime} \mathrm{X}$, 
$\left.{ }^{3} J_{\mathrm{PH}}=12.8 \mathrm{~Hz}\left(J_{\mathrm{MX}}\right), N=8.8 \mathrm{~Hz}, 8 \mathrm{H}, o-\mathrm{H}\right) .{ }^{13} \mathrm{C}-$ NMR (ppm, in $\left.\mathrm{CDCl}_{3}\right): \delta=16.7\left(\mathrm{~d},{ }^{2} \mathrm{~J}_{\mathrm{P}-\mathrm{C}}=1.2\right.$ $\mathrm{Hz}, \mathrm{C} 7) ; 38.8\left(\mathrm{~d}, J_{\mathrm{P}-\mathrm{C}}=51.3 \mathrm{~Hz}, \mathrm{P}-\mathrm{C} 6\right) ; 55.4(\mathrm{~s}$, $\left.\mathrm{O}-\mathrm{C} 5 \mathrm{H}_{3}\right) ; 113.4\left(\mathrm{~d},{ }^{3} \mathrm{~J}_{\mathrm{P}-\mathrm{C}}=13.7 \mathrm{~Hz}, \mathrm{Ar}-\mathrm{C}_{\text {meta }}\right) ; 125.4$ $\left(\mathrm{d}, J_{\mathrm{P}-\mathrm{C}}=77.6 \mathrm{~Hz}, \mathrm{P}-\mathrm{C} 1\right) ; 133.4\left(\mathrm{~d},{ }^{2} J_{\mathrm{P}-\mathrm{C}}=12.3 \mathrm{~Hz}\right.$, Ar- $\left.\mathrm{C}_{\text {ortho }}\right) ; 161.9\left(\mathrm{~d},{ }^{4} \mathrm{~J}_{\mathrm{P}-\mathrm{C}}=3.0 \mathrm{~Hz}, \mathrm{CH}_{3} \mathrm{O}-\mathrm{C} 4\right) .{ }^{31} \mathrm{P}-$ NMR (ppm, in $\mathrm{CDCl}_{3}$ ): $\delta=83.6$. Analysis: Calculated for $\mathrm{C}_{40} \mathrm{H}_{56} \mathrm{Cd}_{2} \mathrm{O}_{4} \mathrm{P}_{4} \mathrm{~S}_{8}\left(1206,1 \mathrm{~g} \mathrm{~mol}^{-1}\right): \mathrm{C}, 39.83 ; \mathrm{H}$, 4.68; S, 21.52\%. Found: C, 39.61; H, 4.53; S, 21.52 \%. LC/MS: $m / z 391.2\left(\left[(\mathrm{~L} 5)_{2} \mathrm{CdS}\right]^{+}, 100 \%\right) ; 602.1$ $\left(\left[\mathrm{Cd}(\mathrm{L} 5)_{2}+\mathrm{Na}\right]^{+}, 12 \%\right) ; 959.8\left(\left[\mathrm{Cd}_{2}(\mathrm{~L} 5)_{3}\right]^{+}, 16 \%\right)$; $1205.6\left(\left[\mathrm{Cd}_{2}(\mathrm{~L} 5)_{4}\right]^{+}, 4 \%\right)$.

\section{$2.3 \quad$ X-ray crystallography}

Single-crystal X-ray diffraction analyses of $\left[\mathrm{Cd}(\mathrm{L} 1)_{2}\right]_{2}$ and $\left[\mathrm{Cd}(\mathrm{L} 2)_{2}\right]_{2}$ were performed on a Bruker Kappa APEXII CCD area-detector diffractometer using Mo $\mathrm{K}_{\alpha}(\lambda=0.71073 \AA)\left(\right.$ for $\left.\left[\mathrm{Cd}(\mathrm{L} 1)_{2}\right]_{2}\right)$ and $\mathrm{Cu} \mathrm{K}_{\alpha}(\lambda=$ $1.5418 \AA$ ) (for $\left[\mathrm{Cd}(\mathrm{L} 2)_{2}\right]_{2}$ ) radiations at a temperature of 100 K. Structures were solved by direct methods ${ }^{18}$ and refined by full-matrix least squares against $F^{2}$ using all data. ${ }^{18}$ All non-H atoms were refined anisotropically. $\mathrm{H}$ atoms were positioned geometrically at distances of $0.93 \AA$ (aromatic $\mathrm{CH}), 0.97 \AA\left(\mathrm{CH}_{2}\right)$ and $0.96 \AA\left(\mathrm{CH}_{3}\right)$ (for $\left.\left[\mathrm{Cd}(\mathrm{L} 1)_{2}\right]_{2}\right)$ and $0.95 \AA$ (aromatic $\mathrm{CH}$ ), $0.99 \AA$ $\left(\mathrm{CH}_{2}\right)$ and $0.98 \AA\left(\mathrm{CH}_{3}\right)$ (for $\left.\left[\mathrm{Cd}(\mathrm{L} 2)_{2}\right]_{2}\right)$ from the parent $\mathrm{C}$ atoms; a riding model was used during the refinement proceses and the $\mathrm{U}_{\text {iso }}(\mathrm{H})$ values were constrained to be $x \mathrm{U}_{\text {eq }}$ (carrier atom), where $x=1.2$ for $\mathrm{CH}$ and $\mathrm{CH}_{2}$, and $x=1.5$ for $\mathrm{CH}_{3}$. Experimental data are given in table 1 .

\section{Results and Discussion}

\subsection{Synthesis and characterization}

In this work, five novel cadmium-DTPA complexes and one new dithiophosphinato ligand $\left(\mathrm{NH}_{4} \mathrm{~L} 2\right)$ were synthesized. LR was reacted with five different Grignard reagents to obtain dithiophosphinic acids. As the dithiophosphinic acids are viscous liquids and difficult

Table 1. Experimental details for $\left[\mathrm{Cd}(\mathrm{L} 1)_{2}\right]_{2}$ and $\left[\mathrm{Cd}(\mathrm{L} 2)_{2}\right]_{2}$.

\begin{tabular}{|c|c|c|}
\hline Compound & {$\left[\mathrm{Cd}(\mathrm{L} 1)_{2}\right]_{2}$} & {$\left[\mathrm{Cd}(\mathrm{L} 2)_{2}\right]_{2}$} \\
\hline Empirical Formula & $\mathrm{C}_{48} \mathrm{H}_{72} \mathrm{Cd}_{2} \mathrm{O}_{4} \mathrm{P}_{4} \mathrm{~S}_{8}$ & $\mathrm{C}_{44} \mathrm{H}_{64} \mathrm{Cd}_{2} \mathrm{O}_{4} \mathrm{P}_{4} \mathrm{~S}_{8}$ \\
\hline Colour/shape & green/block & green/block \\
\hline Formula weight & 1318.24 & 1262.11 \\
\hline Temperature (K) & $100(2)$ & $100(2)$ \\
\hline Radiation, graphite monochr. & $\operatorname{Mo~K}_{\alpha}(\lambda=0.71073)$ & $\mathrm{Cu} \mathrm{K}{ }_{\alpha}(\lambda=1.54178)$ \\
\hline Crystal system & triclinic & triclinic \\
\hline Space group & $\mathrm{P}-1$ & $\mathrm{P}-1$ \\
\hline a, b, c $(\AA)$ & $11.3887(4), 12.2986(4), 12.5052(4)$ & $9.8249(1), 11.6464(1), 13.6449(1)$ \\
\hline$\alpha, \beta, \gamma\left({ }^{\circ}\right)$ & $91.902(1), 107.177(1), 116.112(1)$ & 68.440(1), 83.434(1), 69.380(1) \\
\hline Volume $\left(\AA^{3}\right)$ & $1475.19(8)$ & $1358.85(2)$ \\
\hline Z & 1 & 1 \\
\hline Abs. coefficients $\left(\mathrm{mm}^{-1}\right)$ & 1.151 & 10.560 \\
\hline $\mathrm{D}_{\text {calc }}\left(\mathrm{mg} \mathrm{m}^{-3}\right)$ & 1.484 & 1.542 \\
\hline Max. crystal dim. (mm) & $0.04 \times 0.06 \times 0.08$ & $0.04 \times 0.06 \times 0.08$ \\
\hline$\Theta(\max )\left(^{\circ}\right)$ & 29.19 & 71.08 \\
\hline Reflections measured & 7876 & 5076 \\
\hline Range of h, k, 1 & $-15<\mathrm{h}<14,-16<\mathrm{k}<16,-17<1<17$ & $-12<\mathrm{h}<12,-14<\mathrm{k}<14,-15<\mathrm{l}<16$ \\
\hline Diffractometer/scan & Bruker Kappa & X II / phi and $\mathrm{w}$ \\
\hline No. of reflections with $\mathrm{I}>2 \sigma(\mathrm{I})$ & 7298 & 4902 \\
\hline Corrections applied & Lorentz & rization \\
\hline Computer programs & SHELXS- $97^{18}, \mathrm{SH}$ & L-9 $97^{18}$, ORTEP-3 ${ }^{19}$ \\
\hline Source of scatter. Factors & International Table fo & Ray Crystallography 20 \\
\hline Structure solution & Dire & thods \\
\hline Treatment of hydrogen atoms & Geomet & lculation \\
\hline No. of parameters varied & 298 & 284 \\
\hline GOF & 1.024 & 1.045 \\
\hline $\mathrm{R}=\mathrm{IIF}_{\mathrm{o}} \mathrm{I}-\mathrm{IF}_{\mathrm{c}} \mathrm{II} / \mathrm{IF}_{\mathrm{o}} \mathrm{I}$ & 0.0194 & 0.0222 \\
\hline $\mathrm{R}_{\mathrm{w}}$ & 0.0489 & 0.0589 \\
\hline$(\Delta \rho)_{\max }\left(\mathrm{e} \AA^{-3}\right)$ & 0.499 & 0.709 \\
\hline$(\Delta \rho)_{\min }\left(\mathrm{e} \AA^{-3}\right)$ & -0.318 & -0.396 \\
\hline
\end{tabular}


to purify, they are converted to their ammonium salts (scheme 1).

The $\left[\mathrm{Cd}(\mathrm{L})_{2}\right]_{2}$ complexes were prepared by the reaction of $\mathrm{NH}_{4} \mathrm{~L}$ with cadmium(II) cation (scheme 2).

\subsection{Spectroscopic Studies}

3.2.1 IR and Raman Spectra: In the IR spectra, the asymmetric and symmetric (PS) stretching signals $\left(v_{\text {asym }}\right.$ and $v_{\text {sym }}$ ) of the compounds appear in the regions of $650-616 \mathrm{~cm}^{-1}$ and $552-524 \mathrm{~cm}^{-1}$, respectively. In the Raman spectra, similar signals appear in the regions of $654-632 \mathrm{~cm}^{-1}$ and $555-523 \mathrm{~cm}^{-1}$, respectively. The metal-sulphur stretching give rise to the signals between $200 \mathrm{~cm}^{-1}$ and $400 \mathrm{~cm}^{-1}\left(272-312 \mathrm{~cm}^{-1}\right.$ for IR; 273$310 \mathrm{~cm}^{-1}$ for Raman).
In the IR spectra of $\mathrm{NH}_{4} \mathrm{~L} 2$, the characteristic $v_{\mathrm{N}-\mathrm{H}}$ band is observed at $3183 \mathrm{~cm}^{-1}$; this band is absent in the spectra of the $\left[\mathrm{Cd}(\mathrm{L} 2)_{2}\right]_{2}$ complex, confirming the exclusion of the $\mathrm{NH}_{4}^{+}$ion.

The frequencies of the corresponding signals in the IR and the Raman spectra of the complexes are quite comparable as expected. All these values are in agreement with the literature. ${ }^{14 c, 21}$ The prominent peaks are given in table 2.

3.2.2 Mass Spectra: The mass spectra of the complexes $\left[\mathrm{Cd}(\mathrm{L} 2)_{2}\right]_{2},\left[\mathrm{Cd}(\mathrm{L} 4)_{2}\right]_{2}$ and $\left[\mathrm{Cd}(\mathrm{L} 5)_{2}\right]_{2}$ display molecular ion peaks corresponding to the proposed dinuclear structures. The Mass spectra of all the complexes except $\left[\mathrm{Cd}(\mathrm{L} 3)_{2}\right]_{2}$, display peaks indicating removal of a ligand leaving back a species of the formula

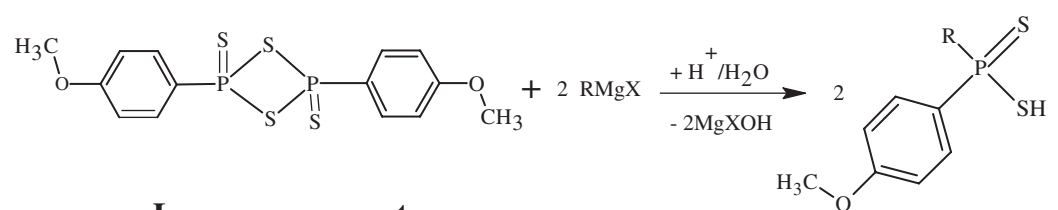

Lawesson reagent

LH<smiles>[R]P(=S)([SH2+])c1ccc(OC)cc1</smiles>

Scheme 1. Synthesis reaction of $\mathrm{NH}_{4} \mathrm{~L}$.

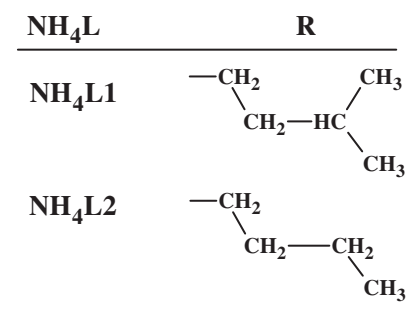

$\mathrm{NH}_{4} \mathrm{L3}$<smiles>CCC(C)C</smiles>

$\mathrm{NH}_{4} \mathbf{L} 4$<smiles>CCC(C)C</smiles>

$\mathrm{NH}_{4} \mathrm{~L} 5$<smiles>CC(C)C</smiles>

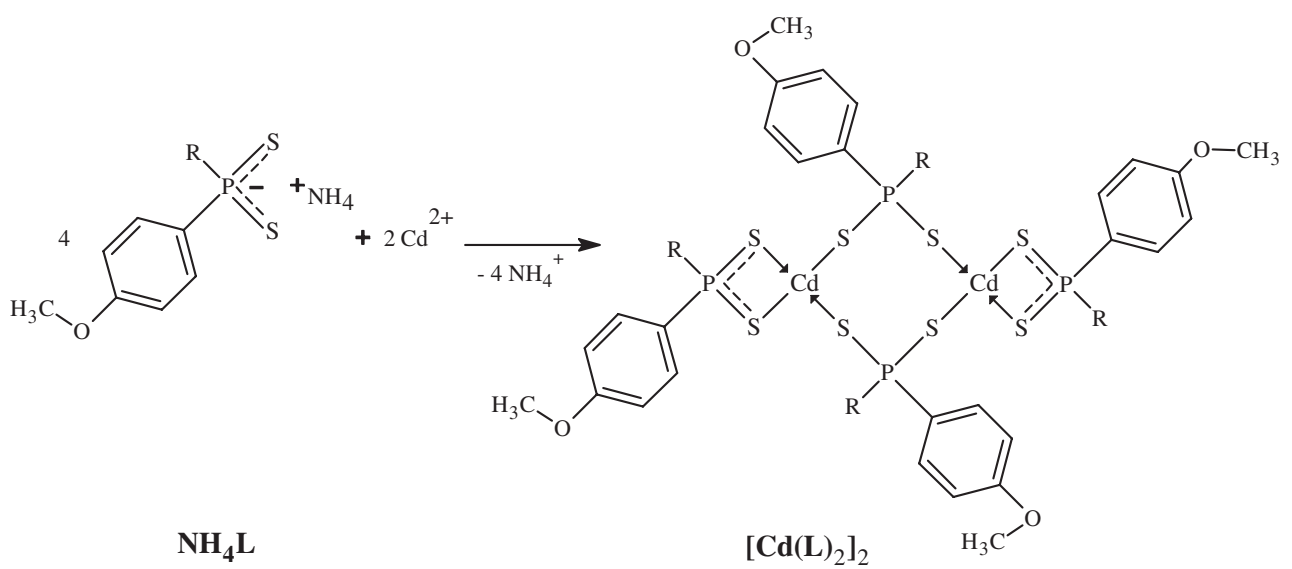

Scheme 2. Synthesis of complexes. 
Table 2. Selected FTIR and Raman (R) data $\left(\mathrm{cm}^{-1}\right)$ assignment of significant bands.

\begin{tabular}{|c|c|c|c|c|c|c|c|c|}
\hline \multirow[t]{2}{*}{ Compound } & \multicolumn{2}{|c|}{$v_{(\mathrm{M}-\mathrm{S})}$} & \multicolumn{2}{|c|}{$v_{(\mathrm{sym})}(\mathrm{PS})$} & \multicolumn{2}{|c|}{$v_{\text {(asym) }}(\mathrm{PS})$} & \multicolumn{2}{|c|}{$v_{(\mathrm{N}-\mathrm{H})}$} \\
\hline & IR & $\mathrm{R}$ & IR & $\mathrm{R}$ & IR & $\mathrm{R}$ & IR & $\mathrm{R}$ \\
\hline$\left[\mathrm{Cd}(\mathrm{L} 1)_{2}\right]_{2}$ & 290 & 289 & 527 & $535 ; 524$ & 638 & $631 ; 632$ & - & - \\
\hline$\left[\mathrm{Cd}(\mathrm{L} 2)_{2}\right]_{2}$ & 312 & 310 & $529 ; 519$ & $542 ; 523$ & $647 ; 635$ & $647 ; 633$ & - & - \\
\hline$\left[\mathrm{Cd}(\mathrm{L} 3)_{2}\right]_{2}$ & 280 & 282 & 526 & $542 ; 525$ & 643 & $652 ; 632$ & - & - \\
\hline$\left[\mathrm{Cd}(\mathrm{L} 4)_{2}\right]_{2}$ & 272 & 273 & $552 ; 532$ & $555 ; 537$ & $650 ; 639$ & $653 ; 633$ & - & - \\
\hline$\left[\mathrm{Cd}(\mathrm{L} 5)_{2}\right]_{2}$ & 274 & 273 & $548 ; 524$ & $549 ; 528$ & $649 ; 640$ & $654 ; 635$ & - & - \\
\hline $\mathrm{NH}_{4} \mathrm{~L} 2$ & - & - & $545 ; 534$ & $549 ; 528$ & $646 ; 616$ & $637 ; 636$ & 3180 & - \\
\hline
\end{tabular}

$\left[\mathrm{Cd}_{2}(\mathrm{~L})_{3}\right]$. The complexes $\left[\mathrm{Cd}(\mathrm{L} 2)_{2}\right]_{2}$ and $\left[\mathrm{Cd}(\mathrm{L} 5)_{2}\right]_{2}$ display Mass-peaks corresponding to the mono-nuclear $\left[\mathrm{Cd}(\mathrm{L} 2)_{2}\right]$ and $\left[\mathrm{Cd}(\mathrm{L} 5)_{2}\right]$ moieties. The natural abundance of metal- as well as phosphorus and sulphur isotopes are observable in the structures of the molecular ion signals. Reminiscent signal structures are

Table 3. Selected bond lengths $(\AA)$ and angles $\left({ }^{\circ}\right)$ for $\left[\mathrm{Cd}(\mathrm{L} 1)_{2}\right]_{2}$ and $\left[\mathrm{Cd}(\mathrm{L} 2)_{2}\right]_{2}$.

\begin{tabular}{|c|c|c|c|}
\hline \multicolumn{2}{|c|}{$\left[\mathrm{Cd}(\mathrm{L} 1)_{2}\right]_{2}$} & \multicolumn{2}{|c|}{$\left[\mathrm{Cd}(\mathrm{L} 2)_{2}\right]_{2}$} \\
\hline Cd1-S1 & $2.5325(3)$ & $\mathrm{Cd} 1-\mathrm{S} 1$ & $2.5460(5)$ \\
\hline Cd1-S2 & 2.6671(3) & Cd1-S2 & $2.6542(4)$ \\
\hline $\mathrm{Cd} 1-\mathrm{S} 3$ & $2.5354(3)$ & $\mathrm{Cd} 1-\mathrm{S} 3^{\mathrm{ii}}$ & $2.5442(5)$ \\
\hline Cd1-S4 & $2.5703(3)$ & Cd1-S4 & $2.5726(4)$ \\
\hline P1-S1 & $2.0252(5)$ & P1-S1 & $2.0253(6)$ \\
\hline P1-S2 & $2.0107(5)$ & P1-S2 & $2.0128(6)$ \\
\hline P1-C1 & $1.8014(13)$ & $\mathrm{P} 1-\mathrm{C} 1$ & $1.8043(19)$ \\
\hline P1-C8 & $1.8161(14)$ & $\mathrm{P} 1-\mathrm{C} 8$ & $1.820(2)$ \\
\hline P2-S3 & $2.0364(4)$ & P2-S3 & $2.0349(6)$ \\
\hline $\mathrm{P} 2-\mathrm{S} 4^{\mathrm{i}}$ & $2.0093(5)$ & P2-S4 & $2.0099(6)$ \\
\hline $\mathrm{P} 2-\mathrm{C} 13$ & $1.7929(13)$ & $\mathrm{P} 2-\mathrm{C} 12$ & $1.7955(19)$ \\
\hline P2-C20 & $1.8181(14)$ & P2-C19 & $1.8166(19)$ \\
\hline $\mathrm{S} 1-\mathrm{Cd} 1-\mathrm{S} 2$ & $79.709(11)$ & $\mathrm{S} 1-\mathrm{Cd} 1-\mathrm{S} 2$ & $79.897(14)$ \\
\hline $\mathrm{S} 1-\mathrm{Cd} 1-\mathrm{S} 3$ & $136.923(11)$ & $\mathrm{S} 1-\mathrm{Cd} 1-\mathrm{S} 3^{\mathrm{ii}}$ & $134.434(15)$ \\
\hline $\mathrm{S} 1-\mathrm{Cd} 1-\mathrm{S} 4$ & $117.464(12)$ & $\mathrm{S} 1-\mathrm{Cd} 1-\mathrm{S} 4$ & $122.969(16)$ \\
\hline $\mathrm{S} 2-\mathrm{Cd} 1-\mathrm{S} 3$ & $108.310(11)$ & $\mathrm{S} 2-\mathrm{Cd} 1-\mathrm{S} 3^{\mathrm{ii}}$ & $107.797(14)$ \\
\hline $\mathrm{S} 2-\mathrm{Cd} 1-\mathrm{S} 4$ & $103.918(11)$ & $\mathrm{S} 2-\mathrm{Cd} 1-\mathrm{S} 4$ & $99.957(14)$ \\
\hline $\mathrm{S} 3-\mathrm{Cd} 1-\mathrm{S} 4$ & $101.913(11)$ & $\mathrm{S} 3-\mathrm{Cd} 1-\mathrm{S} 4^{\mathrm{ii}}$ & $100.388(15)$ \\
\hline $\mathrm{P} 1-\mathrm{S} 1-\mathrm{Cd} 1$ & $85.347(15)$ & P1-S1-Cd1 & $84.86(2)$ \\
\hline P1-S2-Cd1 & $82.104(15)$ & P1-S2-Cd1 & $82.274(19)$ \\
\hline P2-S3-Cd1 & $105.342(16)$ & $\mathrm{P} 2-\mathrm{S} 3-\mathrm{Cd} 1^{\mathrm{ii}}$ & 103.67(2) \\
\hline $\mathrm{P} 2-\mathrm{S} 4-\mathrm{Cd} 1^{\mathrm{i}}$ & $93.481(15)$ & P2-S4-Cd1 & $92.97(2)$ \\
\hline S2-P1-S1 & $111.38(2)$ & S1-P1-S2 & 111.62(3) \\
\hline C1-P1-S1 & $110.23(5)$ & S1-P1-C1 & 111.49(6) \\
\hline C1-P1-S2 & $112.15(5)$ & S1-P1-C8 & $109.54(7)$ \\
\hline C1-P1-C8 & $103.87(6)$ & S2-P1-C1 & 110.68(7) \\
\hline C8-P1-S1 & $109.12(5)$ & S2-P1-C8 & 109.52(7) \\
\hline C8-P1-S2 & $109.80(5)$ & C1-P1-C8 & 103.69(9) \\
\hline $\mathrm{S} 4-\mathrm{P} 2-\mathrm{S} 3^{\mathrm{i}}$ & $113.81(2)$ & S3-P2-S4 & $113.12(3)$ \\
\hline C13-P2-S3 & $110.62(5)$ & S3-P2-C12 & $110.44(6)$ \\
\hline C13-P2-S4 & 111.17(5) & S3-P2-C19 & 106.92(7) \\
\hline C13-P2-C20 & $104.48(6)$ & S4-P2-C12 & $111.35(6)$ \\
\hline C20-P2-S3 & $105.87(5)$ & S4-P2-C19 & 109.20(7) \\
\hline C20-P2-S4 ${ }^{\mathrm{i}}$ & $110.35(5)$ & C12-P2-C19 & 105.41(9) \\
\hline
\end{tabular}

Symmetry codes: (i) $-\mathrm{x},-\mathrm{y},-\mathrm{z}$, (ii) $1-\mathrm{x},-\mathrm{y}, 2-\mathrm{z}$. 
reported for similar compounds. ${ }^{22}$ The patterns of disintegration are compatible with those reported for similar structures. ${ }^{23}$

\subsubsection{NMR Studies}

3.2.3a ${ }^{1} H-N M R$ Spectra: ${ }^{1} \mathrm{H}-\mathrm{NMR}$ spectral data of $\mathrm{NH}_{4} \mathrm{~L} 2$ are given in the Experimental section. The aromatic protons, together with the phosphorus atom constitute an AA'MM'X spin system. The AA'MM' parts (proton signals) display essentially an AMX pattern; because $J_{\mathrm{AM}^{\prime}}$ and $J_{\mathrm{A}^{\prime} \mathrm{M}}$ are close to zero. So the chemical shift assignments of the aromatic protons can be made on the basis of the magnitudes of the coupling constants to phosphorus. Namely, in the compounds, $\left[\mathrm{Cd}(\mathrm{L} 1)_{2}\right]_{2}$, $\left[\mathrm{Cd}(\mathrm{L} 2)_{2}\right]_{2},\left[\mathrm{Cd}(\mathrm{L} 3)_{2}\right]_{2},\left[\mathrm{Cd}(\mathrm{L} 4)_{2}\right]_{2},\left[\mathrm{Cd}(\mathrm{L} 5)_{2}\right]_{2}$ and $\mathrm{L} 2$, the protons in ortho- position to phosphorus display a ${ }^{3} J_{\mathrm{PH}}$ of $\sim 13 \mathrm{~Hz}$ and those meta- to phosphorus a ${ }^{4} J_{\mathrm{PH}}$ of $\sim 2.4 \mathrm{~Hz}$.

The diastreotropic $-\mathrm{CH}_{2}$ - protons at the $\mathrm{C} 8$ position of $\left[\mathrm{Cd}(\mathrm{L} 4)_{2}\right]_{2}$ are partly superimposed but still distinguishable. Similarly the azo-methin proton at the C6 position of the same compound is partly shielded by the intense $-\mathrm{CH}_{3}$ signal at the $\mathrm{C} 7$ position. Similar phenomena are reported for related structures. ${ }^{16}$ The splitting patterns of the signals due to the apical $-\mathrm{CH}_{3}$ groups of the alkyls are all as expected. Similar findings are reported for analogous compounds. ${ }^{24}$

3.2.3b ${ }^{13} C$-NMR Data ${ }^{13} \mathrm{C}$-NMR data of $\mathrm{NH}_{4} \mathrm{~L} 2$ are as given in the Experimental section. Single bond
$J_{\mathrm{P}-\mathrm{C}}$ couplings lie in the range of $77.3-80.3 \mathrm{~Hz}$ for the aromatic carbons and 50.3-52.5 Hz for the aliphatic carbons. Similar trends were reported for structurally related compounds. ${ }^{25}$ The two-bond ${ }^{31} \mathrm{P}_{-}{ }^{13} \mathrm{C}$ coupling for the aromatic carbon atoms ortho- to the phosphorus are observed to be in the narrow range of $12.2-13.1 \mathrm{~Hz}$ in the anisol groups. Interestingly, the three-bond ${ }^{31} \mathrm{P}$ ${ }^{13} \mathrm{C}$ coupling constants of the aromatic carbons are comparatively higher (in the range of $13.7-14.1 \mathrm{~Hz}$ ). ${ }^{2} J_{\mathrm{P}-\mathrm{C}}$ values for the aliphatic carbons appear to change in a wide range. A similar trend is valid for the phosphoruscoupling constants of the aliphatic carbons. For example, in the compound $\left[\mathrm{Cd}(\mathrm{L} 2)_{2}\right]_{2},{ }^{2} J_{\mathrm{P}-\mathrm{C}}=18.2 \mathrm{~Hz}$ whereas in the compound $\left[\mathrm{Cd}(\mathrm{L} 4)_{2}\right]_{2}{ }^{2} J_{\mathrm{P}-\mathrm{C}}=0.0 \mathrm{~Hz}$. ${ }^{3} J_{\mathrm{P}-\mathrm{C}}$ values aliphatic carbons are in the range 1.2-4.3 $\mathrm{Hz}$.

3.2.3c ${ }^{31} \mathrm{P}-$ NMR Spectra The proton-decoupled ${ }^{31} \mathrm{P}-$ NMR spectra of $\left[\mathrm{Cd}(\mathrm{L} 1)_{2}\right]_{2},\left[\mathrm{Cd}(\mathrm{L} 2)_{2}\right]_{2},\left[\mathrm{Cd}(\mathrm{L} 3)_{2}\right]_{2}$, $\left[\mathrm{Cd}(\mathrm{L} 4)_{2}\right]_{2}$ and $\left[\mathrm{Cd}(\mathrm{L} 5)_{2}\right]_{2}$ show singlets at 71.9, 71.5, $70.1,82.5$ and 83,6 ppm, respectively. All these signals appear at 5-8 ppm lower fields compared to those related to the corresponding ligands. ${ }^{12 \mathrm{a}, 16}$

3.2.4 Descriptions of the crystal structures: The X-ray structural determinations of $\left[\mathrm{Cd}(\mathrm{L} 1)_{2}\right]_{2}$ and $\left[\mathrm{Cd}(\mathrm{L} 2)_{2}\right]_{2}$ confirm the assignments of their structures from spectroscopic data. Selected bond lengths and angles are given in table 3 . The molecular structures along with the atom-numbering schemes are depicted in figures 2 and 3 , while the packing diagrams are given in figures 3 and 4 , respectively.

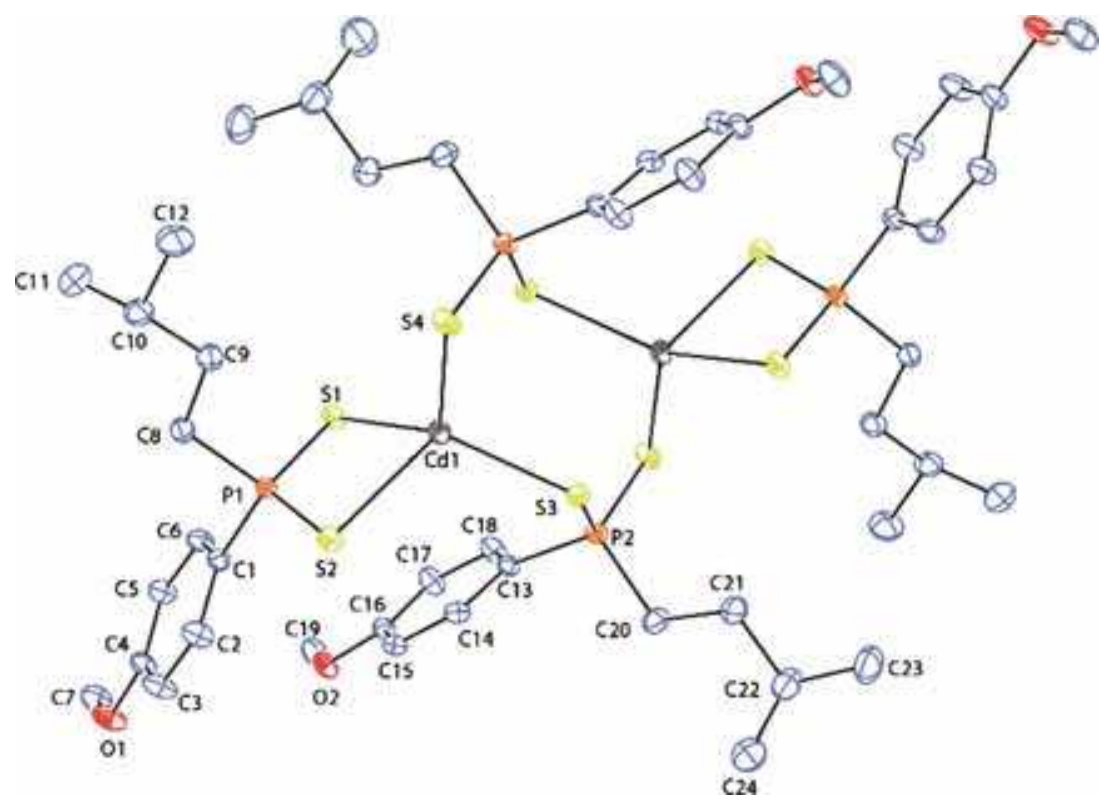

Figure 2. An ORTEP- $3^{19}$ view of $\left[\mathrm{Cd}(\mathrm{L} 1)_{2}\right]_{2}$. The thermal ellipsoids are drawn at the $50 \%$ probability level. 


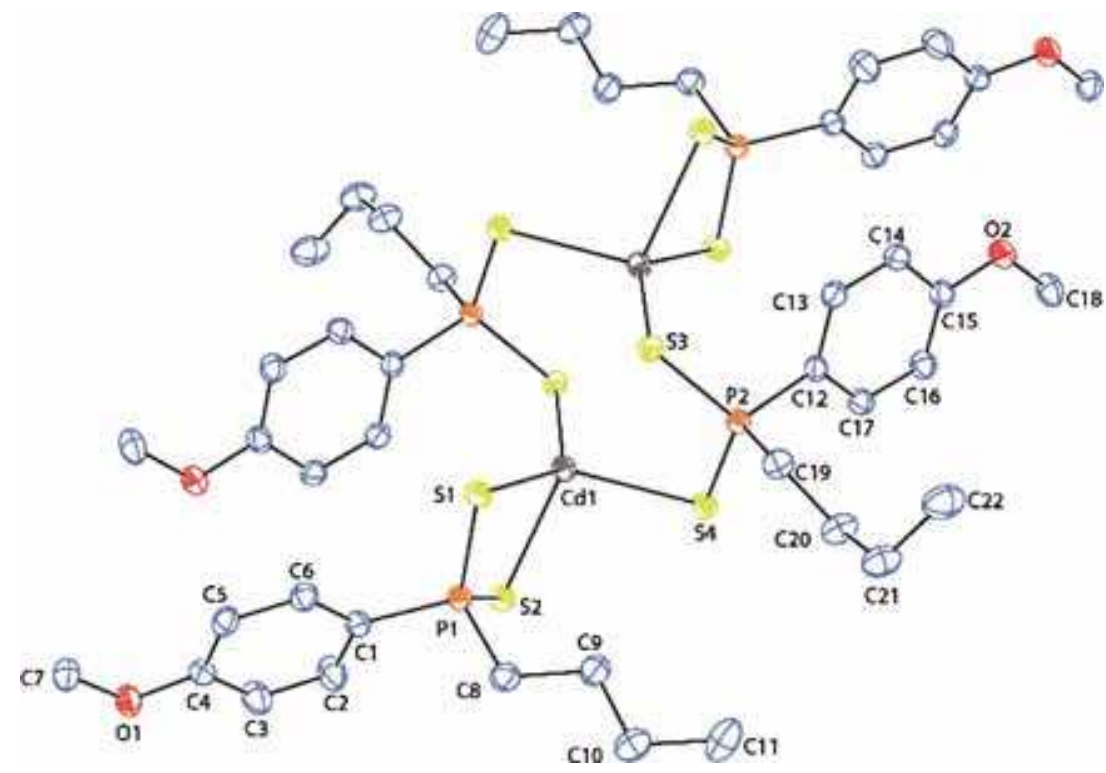

Figure 3. An ORTEP-3 $3^{19}$ view of $\left[\mathrm{Cd}(\mathrm{L} 2)_{2}\right]_{2}$. The thermal ellipsoids are drawn at the $50 \%$ probability level.

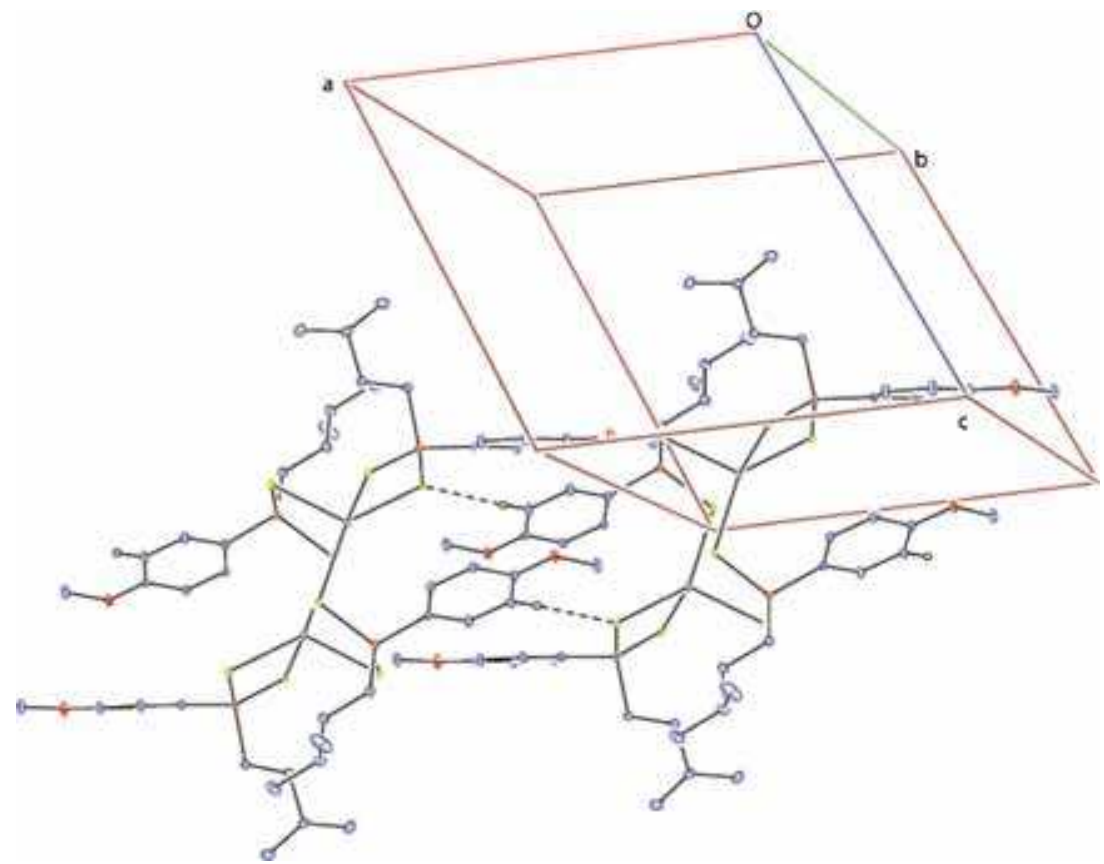

Figure 4. A partial packing diagram of $\left[\mathrm{Cd}(\mathrm{L} 1)_{2}\right]_{2}$. Hydrogen bonds are shown as dashed lines. Hydrogen atoms not involved in hydrogen bonding have been omitted for clarity.

In the centrosymmetric molecules of $\left[\mathrm{Cd}(\mathrm{L} 1)_{2}\right]_{2}$ and $\left[\mathrm{Cd}(\mathrm{L} 2)_{2}\right]_{2}$, the $\mathrm{Cd}$ atoms are coordinated by four $\mathrm{S}$ atoms, while the $\mathrm{P}$ atoms are coordinated by two $\mathrm{S}$ atoms and two $\mathrm{C}$ atoms (figures 2 and 3). The average $\mathrm{Cd}-\mathrm{S}, \mathrm{P}-\mathrm{S}$ and P-C bond lengths and S-P-S and C-P-C bond angles are 2.5763(3) $\AA$, 2.0204(5) $\AA, 1.8071(14)$ $\AA$ and $112.59(2)^{\circ}$ and $104.17(6)^{\circ}$ (for $\left.\left[\mathrm{Cd}(\mathrm{L} 1)_{2}\right]_{2}\right)$ and
2.5792(5) $\mathrm{A}, 2.0207(6) \AA, 1.8091(19) \AA$ and $112.37(3)^{\circ}$ and $104.55(9)^{\circ}$ (for $\left.\left[\mathrm{Cd}(\mathrm{L} 2)_{2}\right]_{2}\right)$, while S-Cd-S and S$\mathrm{P}-\mathrm{C}$ bond angles are in the ranges of $79.709(11)^{\circ}$ $136.923(11)^{\circ}, 105.87(5)^{\circ}-112.15(5)^{\circ}$ (for $\left.\left[\mathrm{Cd}(\mathrm{L} 1)_{2}\right]_{2}\right)$ and $79.897(14)^{\circ}-134.434(15)^{\circ}, 106.92(7)^{\circ}-111.49(6)^{\circ}$ (for $\left[\mathrm{Cd}(\mathrm{L} 2)_{2}\right]_{2}$ ), respectively (table 3 ). The atoms on the $\mathrm{PS}_{2}$ group of the ligand and the central Cd constitute 


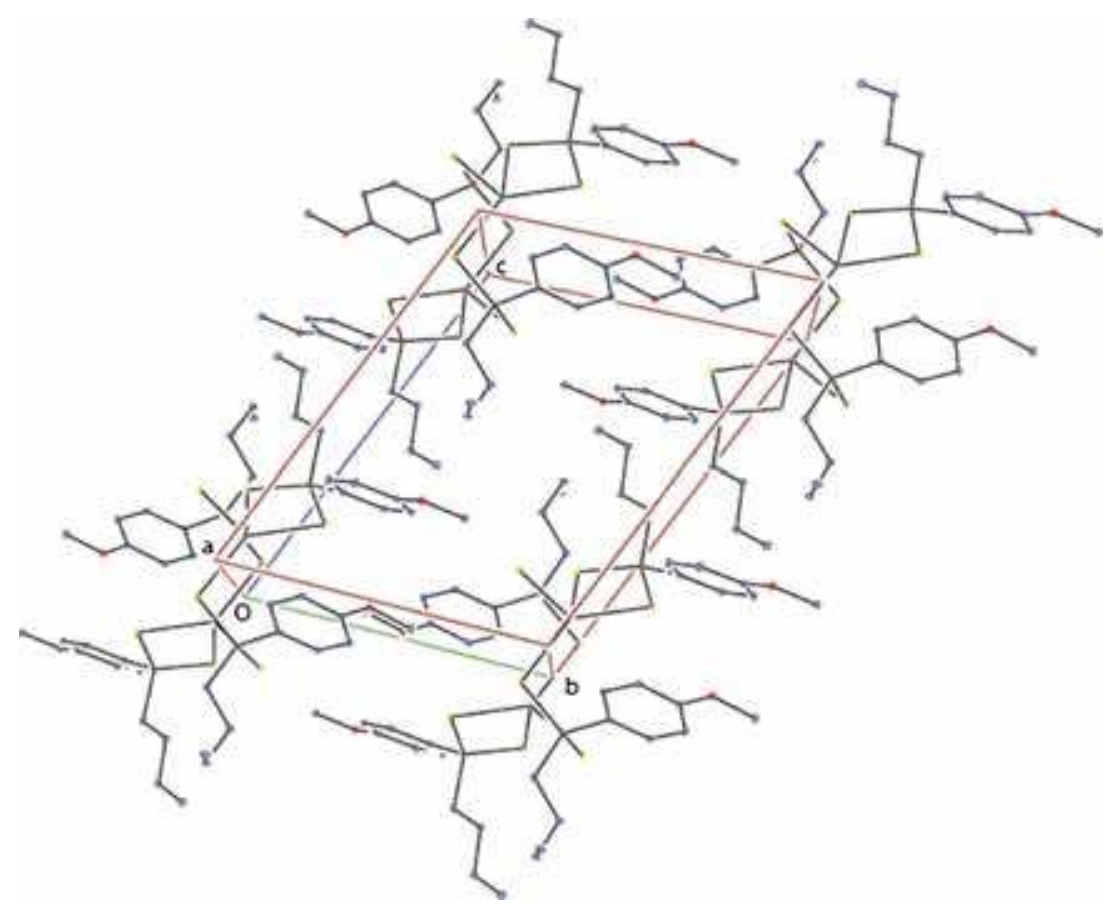

Figure 5. A partial packing diagram of $\left[\mathrm{Cd}(\mathrm{L} 2)_{2}\right]_{2}$. Hydrogen atoms have been omitted for clarity.

a nearly planar geometry. As an indication, the torsion angle $\mathrm{S} 1-\mathrm{Cd} 1-\mathrm{S} 2-\mathrm{P} 1$ is $-7.886(14)^{\circ}$ in $\left[\mathrm{Cd}(\mathrm{L} 1)_{2}\right]_{2}$ and $-7.585(19)^{\circ}$ in $\left[\mathrm{Cd}(\mathrm{L} 2)_{2}\right]_{2}$. In the both compounds, the $\mathrm{Cd}$ atoms have a highly distorted tetrahedral coordination spheres (figures 2 and 3, table 3). This is reflected on S-Cd-S angles. The angle S1-Cd1-S2 is $79.709(11)^{\circ}$ in $\left[\mathrm{Cd}(\mathrm{L} 1)_{2}\right]_{2}$ and $79.897(12)^{\circ}$ in $\left[\mathrm{Cd}(\mathrm{L} 2)_{2}\right]_{2}$; whereas the angle $\mathrm{S} 3-\mathrm{Cd} 1-\mathrm{S} 4$ is $101.913(11)^{\circ}$ and $100.388(15)^{\circ}$ respectively.

The ligand cavities may play important roles in the complexations and metal-ion selectivities. The intramolecular $\mathrm{Cd} 1 \ldots \mathrm{Cd} 1^{\mathrm{i}}[4.1444(2) \AA], \quad \mathrm{S} 3 \ldots \mathrm{S} 3^{\mathrm{i}}$ $\left[\begin{array}{lllll}3.8105(5) & \AA\end{array}\right], \quad \mathrm{S} 4 \quad \ldots \mathrm{S} 4^{\mathrm{i}}\left[6.3172(6) \AA ⿻ 上 丨, \quad \mathrm{P} 2 \ldots \mathrm{P} 2^{\mathrm{i}}\right.$ $\left[\begin{array}{ll}5.6551(5) & \AA\end{array}\right]$ [symmetry code (i) : - x, - y, $\mathrm{z}]\left(\right.$ for $\left.\left[\mathrm{Cd}(\mathrm{L} 1)_{2}\right]_{2}\right)$ and $\mathrm{Cd} 1 \ldots \mathrm{Cd} 1^{\mathrm{i}}[3.8875(2) \AA]$, $\mathrm{S} 3 . . . S 3^{\mathrm{i}}[4.0290(7) \AA], \mathrm{S} 4 \ldots \mathrm{S} 4^{\mathrm{i}}\left[6.1198(7) \AA ̊ 丿 \mathrm{P} 2 . . \mathrm{P} 2^{\mathrm{i}}\right.$ [5.7787(7) Å] [symmetry code (i) : $1-\mathrm{x},-\mathrm{y}, 2-\mathrm{z}$ ] (for $\left.\left[\mathrm{Cd}(\mathrm{L} 2)_{2}\right]_{2}\right)$ distances may indicate the hole sizes of the rings. In the four-membered rings $A(\mathrm{Cd} 1 / \mathrm{S} 1 / \mathrm{S} 2 / \mathrm{P} 1)$, the angles between the $(\mathrm{Cd} 1 / \mathrm{S} 1 / \mathrm{S} 2)$ and $(\mathrm{S} 1 / \mathrm{S} 2 / \mathrm{P} 1)$ planes are $166.10(2)^{\circ}$ (for $\left[\mathrm{Cd}(\mathrm{L} 1)_{2}\right]_{2}$ ) and $166.59(2)^{\circ}$ (for $\left[\mathrm{Cd}(\mathrm{L} 2)_{2}\right]_{2}$ ). Thus, $A$ rings may be called to have twisted-chair conformations. The phenyl rings $B(\mathrm{C} 1-$ C6), $C$ (C13-C18) (for $\left.\left[\mathrm{Cd}(\mathrm{L} 1)_{2}\right]_{2}\right)$ and $B$ (C1-C6), $C$ (C12-C17) (for $\left[\mathrm{Cd}(\mathrm{L} 2)_{2}\right]_{2}$ ) are oriented at dihedral angles of $31.73(5)^{\circ}$ and $24.95(6)^{\circ}$, respectively. In the crystal structures, intermolecular $\mathrm{C} 17-\mathrm{H} 17 . . . \mathrm{S} 1^{\text {ii }}$ interactions $\left(\mathrm{C} 17-\mathrm{H} 17=0.93 \AA, \mathrm{H} 17 \ldots \mathrm{S} 1^{\mathrm{ii}}=2.87 \AA\right.$, $\mathrm{C} 17 \ldots \mathrm{S} 1^{\mathrm{ii}}=3.80 \AA$ and $\mathrm{C} 17-\mathrm{H} 17 \ldots \mathrm{S} 1^{\mathrm{ii}}=177^{\circ}$; symmetry code: (ii) $1-\mathrm{x},-\mathrm{y},-\mathrm{z}$ ) link the molecules into centrosymmetric dimers through $\mathrm{R}_{2}^{2}(16)$ ring motifs ${ }^{26}$ (for $\left[\mathrm{Cd}(\mathrm{L} 1)_{2}\right]_{2}$ ) (figure 4), while the molecules are elongated along the c-axis and stacked along the a-axis (for $\left[\mathrm{Cd}(\mathrm{L} 2)_{2}\right]_{2}$ ) (figure 5).

\section{Conclusions}

The five new dithiophosphinato cadmium(II) complexes and one ligand were prepared. The six novel compounds characterized by, MS, FTIR and RAMAN spectral and elemental analyses. The molecular and crystal structures of the two complexes namely, $\left[\mathrm{Cd}(\mathrm{L} 1)_{2}\right]_{2}$ and $\left[\mathrm{Cd}(\mathrm{L} 2)_{2}\right]_{2}$ were also elucidated by Xray crystallography. The structure of the compounds were analysed by NMR $\left({ }^{1} \mathrm{H},{ }^{13} \mathrm{C},{ }^{31} \mathrm{P}\right)$. For aromatic carbons, the ${ }^{3} J_{\mathrm{P}-\mathrm{C}}$ coupling constants are a few $\mathrm{Hz}$ higher than the ${ }^{2} J_{\mathrm{P}-\mathrm{C}}$ values. ${ }^{31} \mathrm{P}$ chemical shifts the complexes were found to be $5-8 \mathrm{~Hz}$ downfield compared to those of the related dithiophosphonato ligands. X-ray analyses show that the P-S bonds in the complexes are indeed of the same order; in other words, electron delocalization prevails. In the centrosymmetric molecules of $\left[\mathrm{Cd}(\mathrm{L} 1)_{2}\right]_{2}$ and $\left[\mathrm{Cd}(\mathrm{L} 2)_{2}\right]_{2}$, the $\mathrm{Cd}$ atoms are coordinated by four $\mathrm{S}$ atoms, while the $\mathrm{P}$ atoms are coordinated by two $\mathrm{S}$ atoms and two $\mathrm{C}$ atoms. The ligand cavities may play important roles in the complexations and metal-ion selectivities. In the crystal structure of $\left[\mathrm{Cd}(\mathrm{L} 1)_{2}\right]_{2}$, intermolecular C-H...S interactions link the 
molecules into centrosymmetric dimers through $\mathrm{R}_{2}^{2}(16)$ ring motifs.

\section{Supplementary Information}

All additional information relating to the characterization of the complexes, namely, ESI-MS (figure S1), IR spectra (figure S2), Raman spectra (figure S3), NMR $\left({ }^{1} \mathrm{H},{ }^{13} \mathrm{C}\right.$ and $\left.{ }^{31} \mathrm{P}\right)$ spectra (figures $\mathrm{S} 4, \mathrm{~S} 5$ and S6) are available at www.ias.ac.in/chemsci. Crystallographic data (excluding structure factors) have been deposited with the Cambridge Crystallographic Data Centre as the supplementary publication no. 1060403 (for $\left[\mathrm{Cd}(\mathrm{L} 1)_{2}\right]_{2}$, and CCDC 1060404 (for $\left[\mathrm{Cd}(\mathrm{L} 2)_{2}\right]_{2}$ ). Copies of the data can be obtained, free of charge, on application to CCDC, 12 Union Road, Cambridge CB2 1EZ, UK (fax: +44-1223-336033; E-mail: deposit@ccdc.cam.ac.uk).

\section{Acknowledgements}

We gratefully acknowledge the financial assistance of Technical Research Council of Turkey (grant nos. TBAG 114Z091, TUBITAK) and Research and Application Centers of Bozok University (BAP2015 FEF/A153). Our thanks also go to the tutors of the Zurich School of Crystallography, for assistance and guidance with the data collection and structure determination of compound $\left[\mathrm{Cd}(\mathrm{L} 1)_{2}\right]_{2}$ and Prof. Davit Zargarian, Chemistry Department of the Université de Montréal for their support with the X-ray facility for $\left[\mathrm{Cd}(\mathrm{L} 2)_{2}\right]_{2}$.

\section{References}

1. (a) McCleverty J A, Kowalski R S Z, Bailey N A, Mulvaney R and O'Cleirigh D A 1983 J. Chem. Soc. Dalton Trans. 4 627; (b) Satake Y, Kashiwadate K, Iizuka Y, Kouyama T, Katto T and Shiiki Z 1989 US Patent 4826906

2. (a) Pradip K G and Juthika S 2005 Indian J. Chem. Technol. 12 50; (b) Ziyatdinova G K, Budnikov G K, Samigullin A I, Gabdullina, G T, Sofronov A V, Al'metkina L A, Nizamov I S and Cherkasov R A 2010 J. Anal. Chem. 65 1273; (c) Yagishita K and Konishi S 2013 US Patent 8481467

3. (a) Artem'ev A V, Malysheva S F, Gusarova N K, Belogorlova N A, Fedorov S V, Timokhin B V, Smirnov V I and Trofimov B A 2012 Chem. Heterocycl. Comp. 483 448; (b) Michalski Jan, Potrzebowski M and Lopusinski A 1984 US Patent 4470933; (c) Kabra V, Mitharwal S and Singh S 2009 Phosphorus, Sulfur Silicon Relat. Elem. 1842431

4. Bara A C Silvestru C and Haiduc 1991 Anticancer Res. 111651
5. Bellande E, Comazzi V, Laine J, Lecayon M, Pasqualini R, Duatti A and Hoffschir D 1995 Nucl. Med. and Biol. 223315

6. Durckheimer W, Bormann D, Ehlers E, Schrinner E and Heymes R 1988 U.S. Patent 4758556

7. (a) Grigorieva $\mathrm{N}$ A, Pashkov G L, Fleitlikh I Y, Nikiforova L K and Pleshkov M A 2010 Hydrometallurgy 105 82; (b) Xihong H, Guoxin T, Jing C and Linfeng R 2014 Dalton Trans. 43 17352; (c) Wieszczycka K and Zembrzuska J 2014 J. Radioanal. Nucl. Chem. 299709

8. (a) Jing C, Meng W, Xuegang L and Jianchen W 2012 Prog. Nucl. Energ. 54 46; (b) Ghoreishi S M, Ansari K and Ghaziaskar H S 2012 J. Supercrit. Fluids 72288

9. (a) Karakus M and Yilmaz H 2006 Russ. J. Coord. Chem. 326 437; (b) Ilyas S N, Gulnara T G, Dmitriy A T, Almaz R N, Ilnar D N and Rafael A C 2014 Phosphorus, Sulfur Silicon Relat. Elem. 1891354

10. Wagner J, Ciesielski M, Fleckenstein C A, Denecke H, Garlichs F, Ball A and Doering M 2013 Org. Process Res. Dev. 1747

11. (a) Diemert K and Kuchen W 1977 Phosphorus, Sulfur Silicon Relat. Elem. 3 131; (b) Rauhut M M, Currier H A and Wystrach V P 1961 J. Org. Chem. 265133

12. (a) Sağlam E G, Çelik Ö, Yılmaz H and İde S 2010 Transition Met. Chem. 35 399; (b) Yordanov N D, Gochev G, Angelova O and Macicek J 1990 Polyhedron 9 2597; (c) Kuchen W, Metten J and Judat A 1964 Chem. Ber. 97 2306; (d) Cavell R G, Byers W, Day E D and Watkins P M 1972 Inorg. Chem. 11 1598; (e) Daly S R, Keith J M, Batista E R, Boland K S, Kozimor S A, Martin R L and Scott B L 2012 Inorg. Chem. 517551

13. (a) Porta P, Sgamellotti A and Vinciguerra N 1971 Inorg. Chem. 10 541; (b) Pinkerton A A, Ahlers F P, Greiwing H F and Krebs B 1997 lnorg. Chim. Acta 25777

14. (a) Calligaris M, Nardin G and Ripamonti A $1970 \mathrm{~J}$. Chem. Soc. A 714; (b) Cavell R G, Day E D, Byers W and Watkins P M 1972 Inorg. Chem. 11 1759; (c) Casas J S, García-Tasende M S, Sánchez A, Sordo J, Castellano E E and Zukerman-Schpector J 1994 Inorg. Chim. Acta 219115

15. Byrom C, Malik M A, O'Brien P, White A J P and Williams D J 2000 Polyhedron 19211

16. Sağlam E G, Y1lmaz H, Dal H and Hökelek T 2012 Phosphorus, Sulfur Silicon Relat. Elem. 187213

17. Hoffmann H and Schumacher G 1967 Tetrahedron Lett. 312963

18. Sheldrick G M 2008 Acta Cryst. A64, 112

19. Farrugia L J 2012 J. Appl. Cryst. 45, 849

20. International fig for X-Ray Crystallography vol. 41974 (Kynoch Press: Birmingham)

21. (a) Sundee S, Hanlan L and Bernstein J 1975 Inorg. Chem. 14 2012; (b) Czernuszewicz R, Maslowsky E Jr and Nakamoto K 1980 Inorg. Chim. Acta 40199

22. Chakravarty M, Pailloux S, Ouizem S, Smith K A, Duesler E N, Paine R T, Williams N J and Hancock R D 2012 Polyhedron 33327

23. (a) Keck H and Kuchen W 1983 Phosphorus, Sulfur Silicon Relat. Elem. 14 225; (b) Heinz S, Keck H and Kuchen W 1984 Org. Mass Spoctrom. 19 82; (c) Christoph D, Keck H, Kuchen W, Mathow J and Wunderlich H 1987 Inorg. Chim. Acta 132 213; (d) 
Mohan P N, Kuchen W, Keck H and Haegele G J 1977 Inorg. Nuclear Chem. 39833

24. (a) Karakuş M, Yılmaz H, Bulak E and Lonnecke P 2005 Appl. Organometal. Chem. 19 396; (b) M, Yilmaz H, Özcan Y and İde S 2004 Appl. Organometal. Chem. 18 141
25. (a) Ramirez R G, Toscano R A, Silverstu $\mathrm{C}$ and Haiduc I 1996 Polyhedron 21 3857; (b) Przychodzen W 2004 Phosphorus, Sulfur Silicon Relat. Elem. 1791621

26. Bernstein J, Davis R E, Shimoni L and Chang N L 1995 Angew. Chem. Int. Ed. Engl. 341555 